\title{
Oligohydramnios and fetal outcome in term pregnancy
}


amniotic cavity and maternal blood across the wall of the uterus (trans membranous flow) all contribute to the amniotic fluid volume. $^{3}$ In a normal pregnancy, the amount increases by a maximum of $10 \mathrm{ml} /$ day, usually decreases at term, and ranges between 1000-1200ml. ${ }^{3}$ A peak amniotic fluid index is at 32 weeks of gestation, followed by a steady decline until 42 weeks. ${ }^{6}$ This fluid plays a vital role in proper fetal development while its deficiency leads to oligohydramnios sequence or potter sequence (bilateral renal agenesis) characterized by an irregular appearance of the fetus and is not compatible with postnatal life, stillborn showing stigmata of prenatal renal failure and oligohydramnios. ${ }^{7,8}$ Definition of oligohydramnios is based on sonographic criteria oligohydramnios defined as an amniotic fluid index less than $5^{\text {th }}$ centile for gestation, ${ }^{2}$ or amniotic fluid index $\leq 5 \mathrm{~cm},{ }^{9}$ or DVP less than $2 \mathrm{~cm}^{3}$ The onset is usually occurring during the latter part of the third trimester, often when one is overdue. Less commonly occurs in early pregnancy, but if it occurs will have a poor prognosis. ${ }^{2,3}$ The amniotic fluid index alters throughout gestation, but it should be between 10 and $25 \mathrm{~cm}$ in the third trimester. Values below $10 \mathrm{~cm}$ indicate a reduced volume, and those below $5 \mathrm{~cm}$ indicate oligohydramnios. ${ }^{1,2,9,10}$ It complicates approximately 3.9 of pregnancies. $^{3}$ Severe oligohydramnios represented by diminished amniotic fluid volume. The etiological factors of severe oligohydramnios include fetal malformation most often involving the genitourinary tract, chromosomal abnormality, premature rupture of membrane, placental insufficiency, and drugs example NSAID and ACEl. ${ }^{1,3,11}$ This study aimed to determine the association between amniotic fluid index with the mode of delivery and indication of cesarean section and association of amniotic fluid index with the birth outcome.

\section{Methods}

This is a retrospective cohort study carried out at the Maternity Teaching Hospital in Erbil city from $1^{\text {st }}$ March 2018 to the $1^{\text {st }}$ March 2019. It included 300 pregnant women with a singleton pregnancy and gestational age from 37 completed weeks till 42 weeks; 150 women were oligohydramnios and 150 normal. Exclusion criteria included multiple pregnancy, intrauterine death, pregnant women with a ruptured membrane, oligohydramnios less than 37 weeks gestation, and pregnant women with previous two or more cesarean sections. Oligohydramnios patients were recruited from the labor room after detailed history and examination. The data were obtained from patients themselves by direct interview using a specially designed questionnaire after explanation and obtaining written consent from each participant. The questionnaire included name, age, phone number, parity, gestational age at presentation, amniotic fluid index, mode of delivery, indications of cesarean section, and birth outcome. Parity was classified as primigravid, multiparas (1-4), grand multiparas $(\geq 5)$. Indications of the cesarean section included the failure of progress, previous cesarean section, history of infertility, fetal distress, antepartum hemorrhage, and abnormal presentation. The birth outcome included alive, fresh still death, or macerated still death. It also included birth weight, obvious congenital anomaly, admission to neonatal care unit, and APGAR score at the first and fifth minutes. Data were analyzed using the statistical package for the social sciences (version 22). Chi-square test of association was used to compare proportions. Fisher's exact test was used when the expected count of more than $20 \%$ of the cells of the table was less than 5 . The student's test of two independent samples was used to compare two means. A $P$ value of $\leq 0.05$ was considered statistically significant. 


\section{Results}

The total sample size of the study was 300 women, 150 with oligohydramnios (cases), and 150 women with a normal amount of amniotic fluid (the comparison group). Table 1 shows that $18.7 \%$ of the cases aged $\geq 35$ years compared with $8 \%$ of the comparison group $(P=0.007)$.
The proportion of grand-multipara among cases $(32.7 \%)$ was significantly higher than the proportion (13.3\%) among the comparison group $(P<0.001)$. The proportion of cases with a gestational age of 41 weeks $(46.7 \%)$ was significantly higher than the proportion (12\%) among the comparison group $(P<0.001)$

Table 1: Association between amniotic fluid index with age, parity, and gestational age.

\section{Amniotic fluid index}

Normal Oligohydramnios Total

No. (\%) No. (\%) No. $\quad$ (\%) $P$ value

\begin{tabular}{|c|c|c|c|c|c|c|c|}
\hline \multicolumn{8}{|l|}{ Age } \\
\hline$<25$ & 38 & $(25.3)$ & 45 & $(30.0)$ & 83 & $(27.7)$ & \\
\hline $25-34$ & 100 & $(66.7)$ & 77 & $(51.3)$ & 177 & $(59.0)$ & \\
\hline$\geq 35$ & 12 & (8.0) & 28 & $(18.7)$ & 40 & $(13.3)$ & 0.007 \\
\hline \multicolumn{8}{|l|}{ Parity } \\
\hline Primiparity & 49 & $(32.7)$ & 64 & $(42.7)$ & 113 & $(37.7)$ & \\
\hline Multiparity & 81 & $(54.0)$ & 37 & $(24.7)$ & 118 & $(39.3)$ & \\
\hline Grand multiparity & 20 & $(13.3)$ & 49 & $(32.7)$ & 69 & $(23.0)$ & $<0.001$ \\
\hline \multicolumn{8}{|l|}{ Gestational age } \\
\hline 38 & 35 & $(23.3)$ & 29 & $(19.3)$ & 64 & $(21.3)$ & \\
\hline 39 & 97 & $(64.7)$ & 51 & $(34.0)$ & 148 & $(49.3)$ & \\
\hline 41 & 18 & $(12.0)$ & 70 & $(46.7)$ & 88 & $(29.3)$ & $<0.001$ \\
\hline Total & 150 & (100.0) & 150 & (100.0) & 300 & $(100.0)$ & \\
\hline
\end{tabular}


Table 2 shows no significant $(P=0.100)$ difference between the mean age of cases (28.19 years) and that of the comparison group (27.11 years). However, there was a significant difference $(P<0.001)$ between the mean gestational age of cases (39.74 weeks) and that of the comparison group (39.01 weeks). The rate of cesarean section among the cases $(76.7 \%)$ was significantly higher $(P<0.001)$ than the rate among the comparison group $(22.7 \%)$, as presented in Table 3. The table shows that $13 \%$ of those who underwent cesarean section among cases had intra-uterine growth retardation (IUGR), while none of the comparison group had IUGR. The main indications for cesarean section were fetal distress $(27.8 \%$ and $38.2 \%$ for cases and comparison groups respectively), and abnormal presentation $(21.7 \%$ vs. $29.4 \%)$. The other indications are presented in Table 3.

Table 2: Means of age and gestational age by amniotic fluid index.

\begin{tabular}{lccccc}
\hline & \multicolumn{5}{c}{ Amniotic fluid index } \\
& Mean & $( \pm$ SD) & Mean & $( \pm$ OD) & P value \\
\hline Age of patients & 27.11 & $( \pm 4.76)$ & 28.19 & $( \pm 6.52)$ & 0.100 \\
Gestational age & 39.01 & $( \pm 0.85)$ & 39.74 & $( \pm 1.23)$ & $<0.001$ \\
\hline
\end{tabular}

Table 3: Association between amniotic fluid index with the mode of delivery and indications of cesarean section.

\begin{tabular}{lccccccc}
\hline \multicolumn{7}{c}{ Normal } & \multicolumn{2}{c}{ Oligo-hydramnios } & \multicolumn{2}{c}{ Total } & \\
& No. & $(\%)$ & No. & $(\%)$ & No. & (\%) & P value \\
\hline Mode of delivery & & & & & & & \\
Cesarean section & 34 & $(22.7)$ & 115 & $(76.7)$ & 149 & $(49.7)$ & \\
Vaginal delivery & 116 & $(77.3)$ & 35 & $(23.3)$ & 151 & $(50.3)$ & $<0.001$ \\
Total & 150 & $(100.0)$ & 150 & $(100.0)$ & 300 & $(100.0)$ & \\
Indication of Cesarean section & & & & & & & \\
FOP & 8 & $(23.5)$ & 18 & $(15.7)$ & 26 & $(17.4)$ & \\
Previous cesarean section & 1 & $(2.9)$ & 15 & $(13.0)$ & 16 & $(10.7)$ & \\
History of infertility & 2 & $(5.9)$ & 3 & $(2.6)$ & 5 & $(3.4)$ & \\
IUGR & 0 & $(0.0)$ & 15 & $(13.0)$ & 15 & $(10.1)$ & \\
Fetal distress & 13 & $(38.2)$ & 32 & $(27.8)$ & 45 & $(30.2)$ & \\
APH & 0 & $(0.0)$ & 7 & $(6.1)$ & 7 & $(4.7)$ & \\
Abnormal presentation & 10 & $(29.4)$ & 25 & $(21.7)$ & 35 & $(23.5)$ & $0.031^{*}$ \\
Total & 34 & $(100.0)$ & 115 & $(100.0)$ & 149 & $(100.0)$ & \\
\hline
\end{tabular}

*By Fisher's exact test. 
Table 4 shows that $21.3 \%$ of the neonates of the cases were of low birth weight of less than 2500 grams, compared with the neonates of the comparison group $(P<0.001)$. No significant differences were detected between the two groups regarding the development of the congenital anomalies $(P=0.501)$. Around one quarter $(25.3 \%)$ of the neonates of the cases were admitted to the NCU compared to $3.3 \%$ of the neonates of the comparison group $(P<0.001)$. Around one quarter $(24.7 \%)$ of the neonates of the cases had an APGAR score of less than 7 in the first minute of life compared with $10 \%$ of the neonates of the comparison group $(P=0.001)$. All the neonates had an APGAR score of more than 7 in the fifth minute of life.

Table 4: Association between amniotic fluid index with birth outcomes.

\section{Amniotic fluid index}

Normal Oligohydramnios Total
No.
(\%)
No.
(\%)
No.
(\%) $\quad P$ value

\section{Birth weight}

$>2500$

142

(94.7)

118

(78.7)

260

(86.7)

$<2500$

32

(21.3)

40

$<0.001$

\section{Obvious congenital anomaly}

Yes

3

6

(4.0)

9

No

147

(98.0)

144

(96.0)

291

Admission to NCU

Yes

5

145

(96.7)

112

38

(25.3)

43

(74.7)

257

$<0.001$

APGAR in first minute

$\begin{array}{llllllll}>7 & 135 & (90.0) & 113 & (75.3) & 248 & (82.7) & \\ <7 & 15 & (10.0) & 37 & (24.7) & 52 & (17.3) & 0.001\end{array}$

APGAR in fifth minute

\begin{tabular}{llllllll}
$>7$ & 150 & $(100.0)$ & 150 & $(100.0)$ & 300 & $(100.0)$ & NA \\
Total & 150 & $(100.0)$ & 150 & $(100.0)$ & 300 & $(100.0)$ & \\
\hline
\end{tabular}

*By Fisher's exact test 


\section{Discussion}

In this study, maternal age is regarded as a risk factor for developing oligohydramnios. This finding is not similar to the Melamed study, ${ }^{13}$ in which there was no significant difference between studied cases and the comparison group in regard to maternal age. In the current study, the incidence of oligohydramnios increases with advancing gestation age at 41 weeks, which was $(46.7 \%)$ and on the bases that amniotic fluid index alters throughout gestation, ${ }^{2}$ but disagreed with the study of Melamed. ${ }^{13}$ Regarding the parity, the primigravida was more liable to develop oligohydramnios (42.7\%), which agrees with the Jagatia study. ${ }^{14}$ However, it does not agree with the study of Melamed ${ }^{13}$ in which there was no association between parity and oligohydramnios. In this study, the rate of cesarean section in studied cases $(76.7 \%)$ was high if compared with the comparison group (23.3\%). Jandial et al. ${ }^{16}$ agreed with our study in that the rate of cesarean section in the cases group increased compared to the comparison group. In the current study, the indications to perform cesarean section were fetal distress $(27.8 \%)$, abnormal presentation $(21.7 \%)$, and IUGR (defined as sonographically estimated fetal weight below 10 th percentile $)^{13}(13 \%)$. This was similar to the results obtained by Jagatia, ${ }^{14}$ in which the reasons to perform cesarean section were fetal distress and IUGR. In the current study, the incident of low birth weight (less than $2500 \mathrm{gm})^{8}$ was higher in oligohydramnios group (21.3\%) compared to the comparison group, which was statistically highly significant. This finding was in agreement and comparable with the result of Chate, ${ }^{10}(62 \%)$ and another Indian study (Sriya et al.) ${ }^{17}$, in which the occurrence of low birth weight was $58.38 \%$, and Umber et al. ${ }^{18}(36.3 \%)$. APGAR score (Appearance, Pulse, Grimace, Activity, Respiration), is a simple and replicable method to quickly assess the health of newborn children immediately after birth. ${ }^{8}$ In the first minute in this study, there was a significant difference between the two groups. APGAR score $<7$ was more among those with oligohydramnios (24.7\%) compared to $10 \%$ in the comparison group. This result was similar to the result of Umber et al. ${ }^{18}$, Chate $^{10}$ and Guin et al.., ${ }^{9}$ in which there was an increased chance of low APGAR score at $1^{\text {st }}$ minute in the presence of severe oligohydramnios and disagreed with the result of a study done by Jandial et al. ${ }^{16}$ Regarding admission to the neonatal intensive care unit, in this study, there was a significant difference between oligohydramnios group (25.3\%) and the comparison group (3.3\%). Other studies (Chate, ${ }^{10}$ Sriya et al., ${ }^{17}$ Jagatia, ${ }^{14}$ and Jhonson et al. ${ }^{15}$ ) showed a significant increase in the chance of neonatal intensive care unit admission. However, Manzanares et al., ${ }^{12}$ showed no significant difference between the two groups.

\section{Conclusion}

Oligohydramnios is a frequent occurrence and demands intensive fetal surveillance and proper antepartum and intrapartum care. Amniotic fluid index is an important component of biophysical profile scoring. Its assessment in the early third trimester helps to identify women who need more antepartum surveillance so that proper management can be done to improve maternal and perinatal outcomes. Due to intrapartum complications and a high rate of perinatal morbidity and mortality, the cesarean section rates are rising. However, the decision between vaginal delivery and cesarean section should be well balanced to prevent unnecessary maternal morbidity.

\section{Competing interests}

The authors declare no competing interests.

\section{References}

1. Phelan JP, Smith CV, Broussard P, Small M. Amniotic fluid volume assessment using the four-quadrant technique in the pregnancy at 3642 weeks gestation. J Reprod Med 2013; 32 (7):540-2. 
2. Brace RA. Physiology of amniotic fluid volume regulation. Clin Obstet Gynecol 2013; 40(2):2809.

3. Kehl S, Schelkle A, Thomas A, Puhl A, Meqdad K, Tuschy B et al. Single deepest vertical pocket or amniotic fluid index as evaluation test for predicting adverse pregnancy outcome (SAFE trial): A multicenter, open-label, randomized controlled trial. Ultrasound Obstet Gynecol 2016; 47(6):674-9.

4. Oosterhoof H, Haak MC, Arnoudes JG. Acute Maternal Rehydration increases the urine production rate in the near-term human fetus. Am J Obstet Gynecol 2011;183(1):226-9.

5. Sherer DM. A review of amniotic fluid dynamics and the enigma of isolated oligohydramnios. Am J Perinatol 2002;19:253-66.

6. Machado MR, Cecatti JG, Krupa F, Faunds A. Curve of amniotic fluid index measurements in low-risk pregnancy. Acta Obstet Gynecol Scand 2007; 86:37-41.

7. Patrelli TS, Gizzo S, Cosmi E, Carpano MG, Di Gangi S, Pedrazzi $G$ et al. Maternal hydration therapy improves the quantity of amniotic fluid and the pregnancy outcome in third-trimester isolated oligohydramnios: a controlled randomized institutional trial. J Ultrasound Med 2012; 31(2):239-66.

8. Elasandabesee D, Majumdhar S, Sinha S. Obstetricians' attitudes towards isolated oligohydramnios at term. J Obstet and Gynecol 2007; 27(6):574-6.

9. Guin G, Punekar S, Lele A, Khare S. A prospective clinical study of feto-maternal outcome in pregnancies with abnormal liquor volume. J Obstet Gynaecol India 2011; 61:652-5.

10. Chate $P$, Khatri M, Hariharan C. Pregnancy outcome after diagnosis of oligohydramnios at term. Int J Reprod Contracept Obstet Gynecol 2013; 2:23-6.

11. EK S, Andersson A, Johansson A, Kublicas M. Oligohydramnios in uncomplicated pregnancies, beyond 40 completed weeks. A prospective, randomized, pilot study on maternal and neonatal outcomes. Fetal Diagn Ther 2005; 20:182-5.

12. Manzanares S, Carrillo MP, Gonzalez -peran E, Puertas A, Montoya F. Isolated Oligohydramnios in term pregnancy as an indication for induction of labor. J Matern Fetal Neonatal Med 2007; 20:221 -4 .

13. Melamed N, Pardo J, Milstein R, Chen R, Hod M, Yogev $Y$. Perinatal outcome in pregnancies complicated by isolated oligohydramnios diagnosed before 37 weeks of gestation. Am J Obstet Gynecol 2011; 205(241):1-6.

14. JagatiaK, Singh N, Patel S. Maternal and fetal outcome in Oligohydramnios: a study of 100 cases. Int J Med Sci Public Health 2013; 2(3):724 -7 .

15. Johnson JM, Chauhan SP, Ennen CS, Niederhauser A, Magann EF. A comparison of
3 criteria of oligohydramnios in identifying peripartum complications: a secondary analysis. Am J Obstet Gynecol 2007; 197(2):207.e1-7.

16. Jandial C, Gupta S, Sharma S, Gupta M. Perinatal (12) outcome after antepartum diagnosis of oligohydramnios at or beyond 34 weeks of gestation. J K Sci 2007; 9(4):2013-4.

17. Sriya $R$, Singhai $S$. Perinatal outcome in patients with amniotic fluid index $<5 \mathrm{~cm}$. J Obstet Gynaecol India 2001; 51:98-100.

18. Umber A. Perinatal outcome in pregnancies complicated by isolated oligohydramnios at term. Annals KEMU 2009; 15:35-7. 